\title{
Jolly, fit and fat: Should we be singing the "Santa Too Fat Blues"?
}

ABSTRACT
Santa Claus's apparent weight gain, much
chronicled in the popular media, raises the
question of whether his jolly persona could be
at risk. We investigate why Santa remains jolly,
even though he is becoming obese, and what
factors could be keeping him upbeat. Mea-
sures of body mass, mental health and physical
activity were collected from a representative
cohort of Canadian adults surveyed in 1988 and
again 15 years later. Remaining sedentary was
generally associated with a low jolly quotient
JQ). In addition, a "healthy weight" pattern
and remaining "sedentary" was associated
with higher odds of a low JQ than remaining or
becoming obese (from overweight) while stay-
ing active. Although mechanisms for under-
standing how Santa remains active are yet to
be elucidated, we have uncovered a few clues
and conclude that Santa indeed remains jolly
particularly because he is active, and that a
GIFT (graduated intensity fitness training) is
good for combating the "Santa Too Fat Blues."

$\mathrm{R}$ adio stations are again playing upbeat songs $^{1,2}$ about Santa Claus this Christmas, but media images suggest that this seasonal jolliness may be only superficial. With his predilection for the energy-dense cookies provided by millions of children worldwide, Santa's apparent weight gain has been chronicled from earlier thinner depictions of St. Nick to his recent characterization as overweight or obese. Santa's jolly HOHO (Happy, Overweight $\rightarrow$ Happy, Obese) persona could be at risk. Because obesity is strongly related to poor mental health outcomes, such as depression, ${ }^{3,4}$ and US researchers have concluded that Santa's "Jolly Fat" stereotype is likely a myth, ${ }^{5}$ we ask if we should be singing the "Santa Too Fat Blues" (see Appendix I to read the lyrics and listen to the song, available online at www.cmaj.ca/cgi/content/full/175/12/1563/DCI) this Christmas? In particular, we undertook a weighty investigation into why Santa remains jolly, and what might account for his resilience in the face of growing girth.

As it is universally acknowledged that Santa Claus lives at the North Pole in Canada, ${ }^{6}$ we examined prospective Canadian population data to explore whether a HAHA (Happy, Active $\rightarrow$ Healthy, Active lifestyle) factor could balance the $\mathrm{HOHO}$ attributes, and whether this in turn might explain why Santa remains upbeat, even if he is not trim.

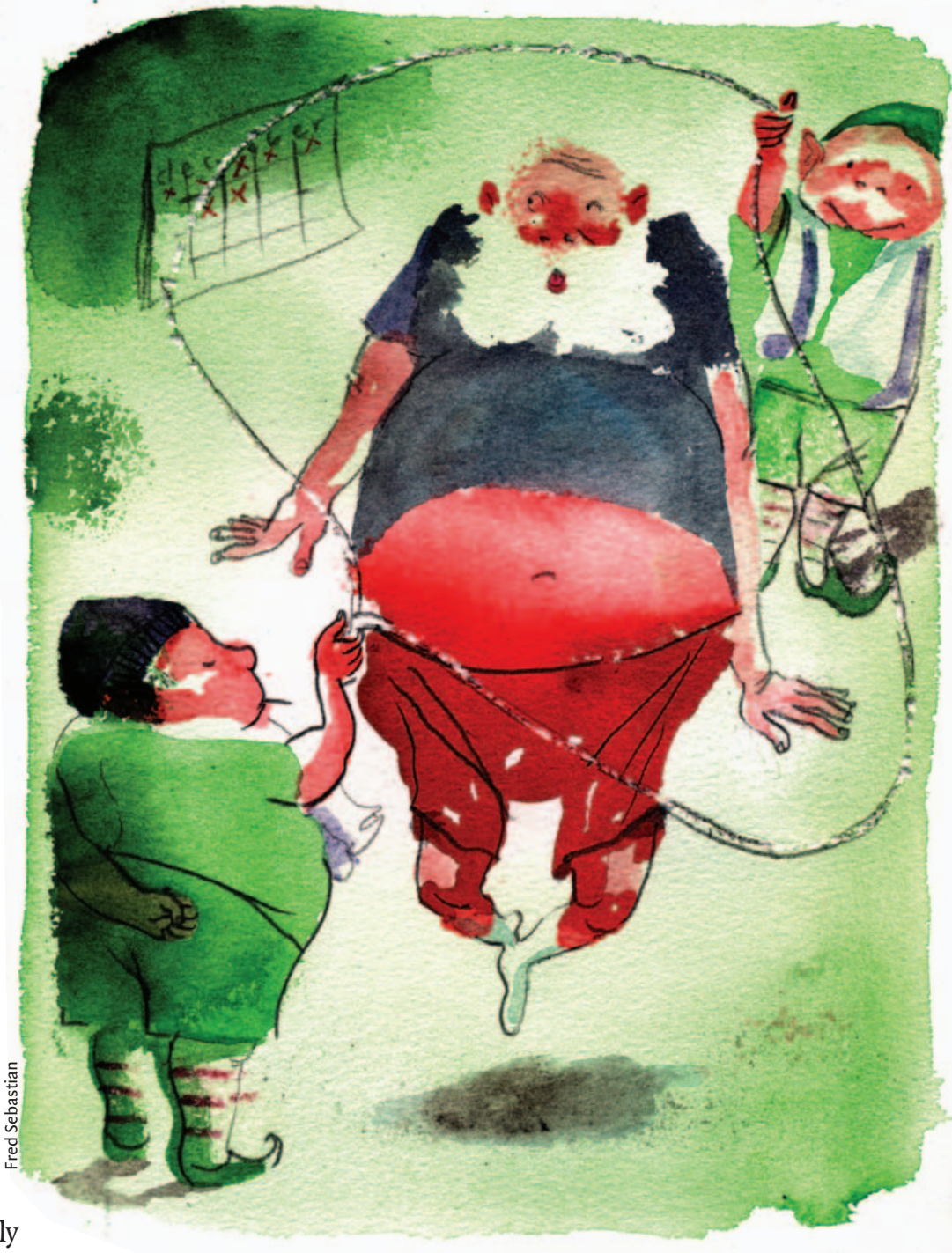

Methods

We surveyed 25II Canadian adults between September 2002 and April $2004^{7}$ who were part of a randomly recruited cohort established in $1988 .^{8}$ Those who were identified as depressed (Center for Epidemiologic Studies-Depression Scale value $>$ I5,$n=$ II4) at baseline were excluded from the study. In total, Iog 8 participants aged $15-69$ years in 1988 completed the 1988 anthropometric measures and answered the questionnaires at baseline and again in 2002-2004. Early examination showed no selection bias among participants.

Jolliness in 2002-2004 was assessed by the standardized mental health subscale of the SF- 36 health survey. ${ }^{9,10}$ A low jolly quotient (JQ) was defined as a below-median score. Height and weight were self-reported in 2002-2004 and 


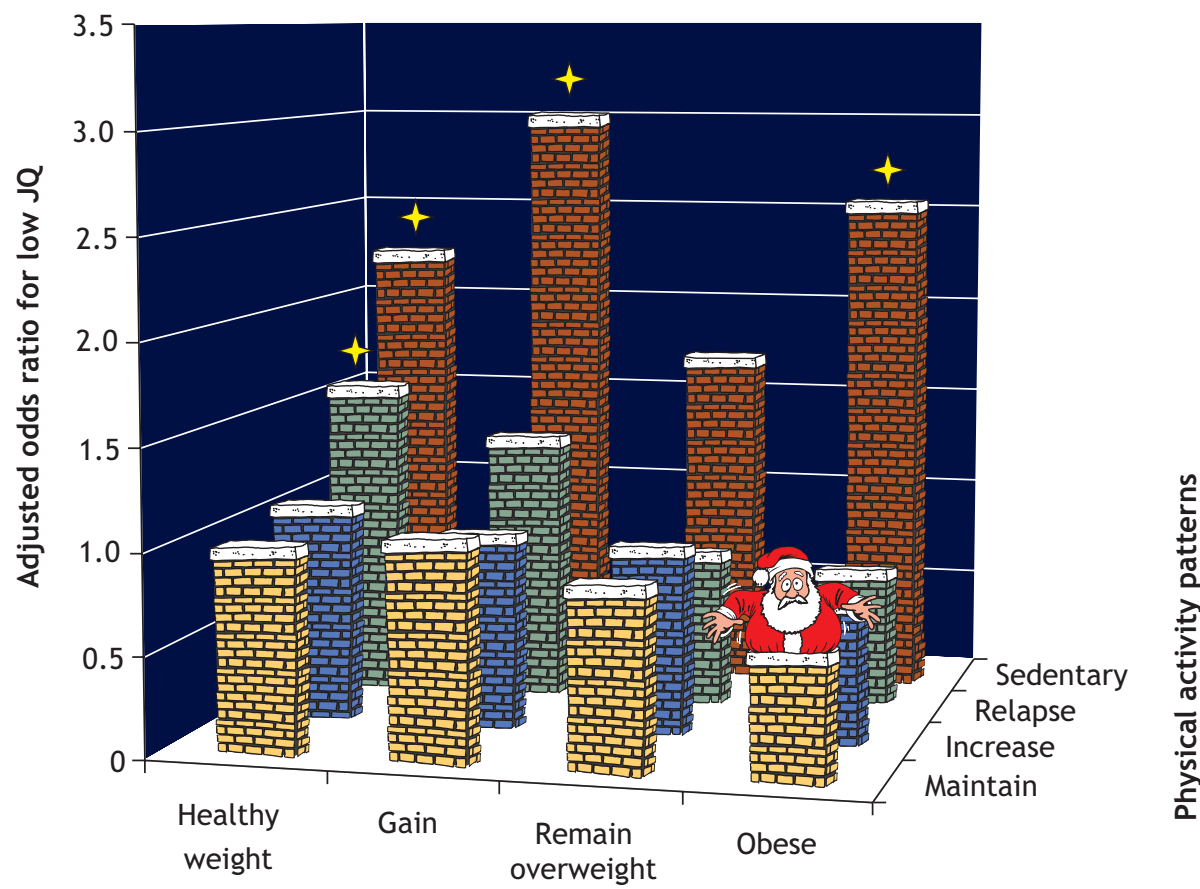

Changes in body mass

$\{p<0.05$

Fig. 1: Adjusted odds of low jolly quotient $(\mathrm{JQ})^{\star}$ in $2002-2004$ by changes in body mass index and physical activity patterns since 1988 in Canadian adults.

*Below median standardized mental health score.

were measured in 1988 using standardized protocols. ${ }^{11}$ Standardized and validated adaptations of the Minnesota Leisure-Time Physical Activity Questionnaire ${ }^{12,13}$ were administered in 1988 and 2002-2004. ${ }^{7}$ This measure was checked for being good or bad, and it was found to be good, for goodness sake. ${ }^{13}$

Body mass index (BMI) was calculated from height and weight and classified as "healthy" $(<25)$, "overweight" (25-29) and "obese" ( $\geq 30)$. Four BMI patterns over the 15year period were categorized as (a) "healthy weight": maintaining or reducing to a healthy weight; (b) "gained": becoming overweight/obese from a healthy weight; (c) remaining "overweight," including reduced from obese; and (d) remaining "obese" or became obese from overweight. An energy expenditure score was calculated by summing the products of the metabolic cost of each type of physical activity reported, its duration in hours and the average number of occasions per week..$^{13}$ Levels were defined in kilocalories per kilogram of body weight per day (KKD) as "sufficient" $(\geq \mathrm{I} .5$ $\mathrm{KKD}$, equivalent to dancing and prancing about 15 minutes daily); "low" (0.5-I.4 KKD); and "sedentary" (< $0.5 \mathrm{KKD})$. Four patterns of physical activity over the I5-year period were created: (a) "maintain": remaining sufficiently active; (b) "increase": increasing from low or sedentary to sufficiently active; (c) "relapse": decreasing to low or low activity at both times; and (d) "sedentary": either reducing to or remaining sedentary.

Adjusted odds ratios of a low JQ were predicted from the patterns of BMI and physical activity over the 15 -year period, controlling for age and sex. The healthiest combination, "healthy weight" and "maintain," served as the referent.

\section{Results}

Fig. I depicts the relation between differing patterns of body mass and physical activity over the 15 -year period. Table I indicates that following a sedentary lifestyle in both 1988 and 2002-2004 is generally associated with an increased odds of a low JQ regardless of changes in body mass. Indeed, having a "healthy weight" pattern with a long-term "sedentary" lifestyle was associated with higher odds of a low JQ than was remaining or becoming obese (from overweight), while maintaining an active lifestyle.

\section{Interpretation}

This analysis helps to explain why an active, albeit overweight, Santa Claus is as likely to be as jolly as a "healthy weight" adult. The mechanisms for understanding how Santa remains active have yet to be elucidated. Could Santa have managed to buck the odds, with the help of activity-supporting reindeer?

Consider these clues. It is undoubtedly the team leader who coaches the reindeer for the annual Reindeer Games. ${ }^{14}$ This would require at least a low level of activity. However, to have the stamina to travel at light speed, ${ }^{15}$ Santa must follow a strict fitness regime like other racing drivers. ${ }^{16}$ Furthermore, ELF 
Table 1: Adjusted odds of low jolly quotient (JQ) in 2002-2004 by changes in body mass and activity patterns since 1988 in 1098 Canadian adults

\begin{tabular}{|c|c|c|c|c|}
\hline Change in body mass and activity pattern, 1988 to $2002-2004$ & $\begin{array}{l}\text { No. of } \\
\text { adults }\end{array}$ & $\mathrm{AOR}^{*}$ & $95 \% \mathrm{Cl}$ & $p$ value \\
\hline \multicolumn{5}{|l|}{ Healthy weight pattern $\dagger$} \\
\hline Maintained activity & 224 & & Reference & \\
\hline Increased activity & 88 & 1.08 & $0.66-1.79$ & 0.75 \\
\hline Relapsed (including remained at a low level of activity) & 112 & 1.59 & $1.01-2.52$ & 0.047 \\
\hline Remained sedentary & 54 & 2.25 & $1.22-4.14$ & 0.009 \\
\hline \multicolumn{5}{|l|}{ Gained weight } \\
\hline Maintained activity & 76 & 1.07 & $0.63-1.82$ & 0.80 \\
\hline Increased activity & 27 & 0.97 & $0.43-2.20$ & 0.95 \\
\hline Relapsed (including remained at a low level of activity) & 41 & 1.35 & $0.69-2.64$ & 0.38 \\
\hline Remained sedentary & 19 & 3.00 & $1.10-8.19$ & 0.032 \\
\hline \multicolumn{5}{|l|}{ Remained overweight $\neq$} \\
\hline Maintained activity & 130 & 0.89 & $0.56-1.41$ & 0.62 \\
\hline Increased activity & 55 & 0.94 & $0.51-1.73$ & 0.84 \\
\hline Relapsed (including remained at a low level of activity) & 65 & 0.77 & $0.43-1.39$ & 0.39 \\
\hline Remained sedentary & 34 & 1.71 & $0.82-3.54$ & 0.15 \\
\hline \multicolumn{5}{|l|}{ Obese§ } \\
\hline Maintained activity & 54 & 0.62 & $0.32-1.18$ & 0.14 \\
\hline Increased activity & 31 & 0.69 & $0.31-1.54$ & 0.37 \\
\hline Relapsed (including remained at a low level of activity) & 49 & 0.68 & $0.35-1.31$ & 0.25 \\
\hline Remained sedentary & 39 & 2.55 & $1.26-5.19$ & 0.010 \\
\hline
\end{tabular}

Note: $\mathrm{AOR}=$ adjusted odds ratio, $\mathrm{Cl}=$ confidence interval.

*Adjusted odds of low JQ (below median health score) among the asymptomatic population at baseline, controlling for age group and sex. †Retained or achieved a healthy weight.

†Remained overweight or reduced from obese to overweight.

SRemained obese or increased to obese from overweight.

(Enhanced Lung Function) appears to be associated with GIFT (Graduated Intensity Fitness Training) programs. Finally, the ability to race from rooftop to rooftop and contort through chimneys of all shapes and sizes indicates that although Santa may be cheerful, he is also likely to practise JOLLY (JOgging and Life-Long Yoga). This is the secret of Santa's persona.

We conclude that Santa Claus indeed remains jolly, because he is active. The carousing Christmas corollary for us and our coronaries is to ask Santa to bring us all the special GIFT of an active lifestyle. It is good medicine to combat the "Santa Too Fat Blues."

\section{Cora L. Craig}

Canadian Fitness and Lifestyle Research Institute

Ottawa, Ont.

Faculty of Applied Health

University of Waterloo

Waterloo, Ont.

\section{Adrian Bauman}

Centre for Physical Activity and Health

School of Public Health

University of Sydney

Sydney, Australia

Canadian Fitness and Lifestyle Research Institute

Ottawa, Ont.

\section{Philayrath Phongsavan}

Centre for Physical Activity and Health

School of Public Health

University of Sydney

Sydney, Australia

Thomas Stephens

Thomas Stephens and Associates

Manotick, Ont.

\section{Stephen J. Harris}

Retired, Guitarist

Ottawa, Ont.

This article was peer reviewed by some short but fit people found chasing a wayward reindeer at the local shopping mall.

Competing interests: Plenty. Everyone has a stake in the well-being of Santa.

Details of funding and independence of authors: Milk and cookies donated by millions of people worldwide. The current wave of data collection for the study was funded by the Social Sciences and Humanities Research Council of Canada and the Canadian Institutes of Health Research (Strategic Joint Initiative Grant on Society, Culture and the Health of Canadians II Grant No. 839-2000-I032). The I988 data collection was funded by the Public Health Agency of Canada (formerly Fitness Canada), National Health Research Development Program Health Canada and Campbell Soup Company Ltd. Although all authors are independent of the funding bodies, the Canadian Fitness and Lifestyle Research Institute receives funding on an annual basis from the Public Health Agency of Canada. 
Acknowledgements: We sincerely thank our following international colleagues for their insights into Christmas lore: R. Petteri (Finland), D. Dasher and D. Dancer (United Kingdom), P. Prjón and V. Refalæch (Iceland); C. Komma and C. Cupido (Sweden), and D. Donner and B. Blixten (the Netherlands).

Ethical approval: This study was approved by the Ethics of Belief Committee, North Pole, and the Ethics Review Board, Université de Montréal, Montréal, Que. (seriously).

Signed consent: All participants (except Santa) signed informed consent forms according to the requirements of the Canadian Fitness and Lifestyle Research Institute and the Ethics Review Board of the Universite de Montréal.

\section{REFERENCES}

I. The-North-Pole. Jolly old Saint Nicolas. Available: www.the-north-pole.com/carols /jollyold.html (accessed 2006 Oct 2).

2. Autrey G, Haldeman O. Here comes Santa Claus. 1947. Available: www.the-northpole.com/carols/herecomes.html (accessed 2006 Oct 2).

3. Roberts RE, Deleger S, Strawbridge WJ, et al. Prospective association between obesity and depression: evidence from the Alameda County Study. Int J Obes Relat Metab Disord 2003;27:514-2I.

4. Carpenter KM, Hasin DS, Allison DB, et al. Relationships between obesity and DSM-IV Major Depressive Disorder, suicide ideation, and suicide attempts: results from a general population study. Am J Public Health 2000;90:25I-7.

5. Sci/Tech News Staff. Softpedia News. The "Jolly Fat" stereotype is myth: even
Santa Claus gets depressed. July 6, 2006. Available: http://news.softpedia.com /news/The-039-Jolly-Fat-039-Stereotype-is-Myth-Even-Santa-Claus-Gets-Depressed28853.shtml (accessed 2006 Oct 2).

6. Canada Post. Write to Santa Claus, North Pole, Canada, HOH OHO. Available: www.canadapost.ca/corporate/about/newsroom/pr/archive-e.asp?prid=I030 (accessed 2006 Oct 2).

7. Craig CL, Gauvin L, Cragg SE, et al. Towards a social epidemiological perspective of physical activity and health: the aims, design and methods of the Physical Activity Longitudinal Study (PALS). JPAH 2005;3:272-84.

8. Stephens TM, Craig CL. The well-being of Canadians: highlights of the Ig88 Campbell's Survey. Ottawa: Canadian Fitness and Lifestyle Research Institute; I990.

9. McHorney CA, Ware JE, Raczek AE. The MOS 36 -item Short-Form Health Survey (SF-36r):III tests of data quality, scaling assumptions and reliability across diverse patient groups. Med Care I994;32:40-66.

Io. Ware JE, Kosinski M, Dewey JE. How to score version 2 of the SF-36 Health Survey. Lincoln, RI: QualityMetric Inc.; 2000.

II. Fitness Canada. Canadian Standardized Test of Fitness (CSTF): Operations Manual. 3rd Edition. Ottawa: Minister of Supply and Services; 1987.

I2. Taylor HL, Jacobs DR Jr, Shucker B, et al. A questionnaire for the assessment of leisure time physical activities. J Chronic Dis I978;31:74I-55

I3. Craig CL, Russell SJ, Cameron C. Reliability and validity of Canada's Physical Activity Monitor for assessing trends. Med Sci Sports Exerc 2002;34:1462-7.

I4. Marks J. Rudolph the red-nosed reindeer. I949. Available: www.the-north-pole .com/carols/rudolph.html (accessed 2006 Oct 2).

I5. See NORAD information on the history and methods used to track Santa Claus. Available: www.noradsanta.org/en/how_we_do_it.php (accessed 2006 Oct 2).

I6. See Formula I fitness requirements for drivers. Available: www.formular.com/insight /technicalinfo/II/980.html (accessed 2006 Oct 2).
Every year, we distribute more than 1.5 million copies of $C M A J$ to Canadian physicians and health professionals around the world.

\section{CMAJ}

for the best of Canadian medicine

Orders and information:

\section{CMA Member Service Centre}

tel $888855-2555$ or

$613731-8610 \times 2307$

fax $613236-8864$

cmamsc@cma.ca
Chaque année, nous distribuons de par le monde plus de 1,5 million d'exemplaires du $J A M C$ aux médecins et aux professionnels de la santé.

\section{Le JAMC}

tout simplement la crème de la médecine canadienne

Abonnements et demandes de renseignements :

Centre des services aux membres de l'AMC tél 888 855-2555 ou

$613731-8610 \times 2307$

fax $613236-8864$

cmamsc@cma.ca

\section{ASSOCIATION

